\title{
Differences in Virulence Between Legionella pneumophila Isolates From Human and Non-human Sources Determined in Galleria mellonella Infection Model
}

\begin{abstract}
Patrícia S. Sousa ${ }^{1}$, Inês N. Silva ${ }^{2}$, Leonilde M. Moreira ${ }^{2,3}$, António Veríssimo ${ }^{1,4}$ and Joana Costa ${ }^{1,4 *}$

${ }^{1}$ Department of Life Sciences, University of Coimbra, Coimbra, Portugal, ${ }^{2}$ Institute for Bioengineering and Biosciences, Instituto Superior Técnico, University of Lisbon, Lisbon, Portugal, ${ }^{3}$ Department of Bioengineering, IST, University of Lisbon, Lisbon, Portugal, ${ }^{4}$ Centre for Functional Ecology - Science for People \& the Planet, Department of Life Sciences, University of Coimbra, Coimbra, Portugal
\end{abstract}

Legionella pneumophila is a ubiquitous bacterium in freshwater environments and in many man-made water systems capable of inducing pneumonia in humans. Despite its ubiquitous character most studies on L. pneumophila virulence focused on clinical strains and isolates from man-made environments, so little is known about the nature and extent of virulence variation in strains isolated from natural environments. It has been established that clinical isolates are less diverse than man-made and natural environmental strains, suggesting that only a subset of environmental isolates is specially adapted to infect humans. In this work we intended to determine if unrelated $L$. pneumophila strains, isolated from different environments and with distinct virulence-related genetic backgrounds, displayed differences in virulence, using the Wax Moth Galleria mellonella infection model. We found that all tested strains were pathogenic in G. mellonella, regardless of their origin. Indeed, a panoply of virulence-related phenotypes was observed sustaining the existence of significant differences on the ability of $L$. pneumophila strains to induce disease. Taken together our results suggest that the occurrence of human infection is not related with the increased capability of some strains to induce disease since we also found a concentration threshold above which L. pneumophila strains are equally able to cause disease. In addition, no link could be established between the sequence-type (ST) and L. pneumophila pathogenicity. We envision that in man-made water distribution systems environmental filtering selection and biotic competition acts structuring $L$. pneumophila populations by selecting more resilient and adapted strains that can rise to high concentration if no control measures are implemented. Therefore, public health strategies based on the sequence based typing (STB) scheme analysis should take into account that the major disease-associated clones of $L$. pneumophila were not related with higher virulence in $G$. mellonella infection model, and that potential variability of virulence-related phenotypes was found within the same ST.

Keywords: Legionella pneumophila, Galleria mellonella, virulence, environmental selection, natural reservoirs, man-made environments, disease 


\section{INTRODUCTION}

Legionella pneumophila is a facultative intracellular Gramnegative bacterium ubiquitous in both freshwater environments and in many man-made water systems known for its ability to cause disease in humans (Fields, 2008; Newton et al., 2010; Cunha et al., 2016). Eukaryotic phagocytes feed on L. pneumophila, so the bacterium's survival and spread relies on the capability to take over the predator cellular mechanism (Fields, 2008; Declerck, 2010; Abdelhady and Garduño, 2013; Richards et al., 2013). Indeed, the machinery underlying Legionella virulence is the outcome of a significant selective pressure resulting in a survival mechanism of trained resilience to protozoa phagocytosis (Moliner et al., 2010; Allombert et al., 2013; Richards et al., 2013; Boamah et al., 2017; Oliva et al., 2018).

L. pneumophila infection proceeds by a conserved mechanism in unrelated phagocytes, with the formation of a replicative niche, which in turn is a prerequisite for the development of Legionnaires' disease (Isberg et al., 2009; Taylor et al., 2009; Al-Quadan et al., 2012; Hoffmann et al., 2014). In humans, Legionella reaches the lungs after inhalation of contaminated aerosol droplets where a parallel procedure allows the bacterium to takeover another phagocyte, lung-based macrophages, leading to infection (Muder et al., 1986; Hubber and Roy, 2010; Newton et al., 2010; Buchrieser, 2011; Finsel and Hilbi, 2015). Recent evidences suggested the rare possibility of person-to-person transmission of L. pneumophila under unique environmental conditions (Borges et al., 2016; Correia et al., 2016).

The evolutive dead-end nature of Legionella infection in humans implies that the long-term co-evolution with free-living protozoa hosts provides the primary evolutionary pressure for acquisition and maintenance of virulence factors (Moliner et al., 2010; Newton et al., 2010; Coscollá et al., 2011; Luo, 2011; AlQuadan et al., 2012; Sánchez-Busó et al., 2014). Indeed, protozoa act as a gene pool enabling various microorganisms to evolve by gene acquisition and loss, adapting to an intra-protozoa lifestyle or progress into new forms (Thomas and Greub, 2010; GomezValero et al., 2011; Al-Quadan et al., 2012; Boamah et al., 2017; Oliva et al., 2018). In accordance, recombination, horizontal gene transfer and convergent evolution play an important role (Costa et al., 2010, 2014; Gomez-Valero and Buchrieser, 2013) in assembling a highly plastic L. pneumophila genome (Coscollá et al., 2011; Sánchez-Busó et al., 2014).

Regardless of the highly diverse populations of Legionella found in natural environments (Veríssimo et al., 1991; Marrão et al., 1993; Costa et al., 2005; Peabody et al., 2017; Zhang et al., 2017; Cassell et al., 2018), L. pneumophila serogroup 1 (SG1) is responsible for $>84 \%$ of the reported cases of disease worldwide (Yu et al., 2002; Harrison et al., 2009; Beauté et al., 2013; Beauté and Network on behalf of the E. L. D. S., 2017). Curiously, the presence of L. pneumophila SG1 in the environment (44\%) differs significantly from the clinical L. pneumophila SG1 strains (84\%) (Harrison et al., 2007; Kozak et al., 2009; David et al., 2016). Nevertheless, it remains unclear why specific L. pneumophila strains are more connected with disease since it could be related with intrinsic virulence or they could merely be present in great numbers in the environments that support their dispersal to human's (Gomez-Valero et al., 2014; McAdam et al., 2014).

As expected, and despite the ubiquitous character of Legionella spp. in fresh water (Fields, 2008; Peabody et al., 2017; Cassell et al., 2018), most studies on the L. pneumophila distribution focused on man-made aquatic environments (air conditioningsystems, potable water distribution systems, public fountains, and plumbing fixtures) in an attempt to establish an epidemiological link between putative environmental sources and clinical isolates (Hyland et al., 2008; Nygård et al., 2008; Sanchez et al., 2008; Phin et al., 2014; Hoisington et al., 2015; van Heijnsbergen et al., 2015, 2016). Several studies support that L. pneumophila clinical isolates are genetically distinct than man-made and natural environmental isolates (Costa et al., 2005, 2010, 2012, 2014; Harrison et al., 2007, 2009; Coscolla and GonzalezCandelas, 2009). These evidences sustain the hypothesis that only a specific subset of environmental isolates are able to cause disease in humans (Harrison et al., 2007; Coscolla and GonzalezCandelas, 2009; David et al., 2016). Indeed, virtually nothing is known about the nature and extent of virulence-related variation in L. pneumophila strains isolated from natural environments (Costa et al., 2005, 2010, 2014), namely streams, rivers, lakes, boreholes, contrarily to the vast number of virulence-related studies performed with L. pneumophila disease-related isolates (Hyland et al., 2008; Nygård et al., 2008; Sanchez et al., 2008; Phin et al., 2014; Hoisington et al., 2015; van Heijnsbergen et al., 2015, 2016).

The use of larvae of the greater Wax Moth Galleria mellonella as an infection model has increased in the last years (Ramarao et al., 2012; Cook and McArthur, 2013; Tsai et al., 2016) since mammalian models imply high costs, ethical constrains and special training. G. mellonella has been used as an alternative model of infection after establishing a correlation between virulence in insect and mammalian models for a wide range of human pathogens providing useful information on their pathogenicity; namely for Staphylococcus aureus, Proteus vulgaris, Serratia marcescens, Pseudomonas aeruginosa, Listeria monocytogenes, Streptococcus suis, or Enterococcus faecalis (Junqueira, 2012; Cook and McArthur, 2013; Eisenman, 2015; Champion et al., 2016; Tsai et al., 2016; Velikova et al., 2016; Wojda, 2017). In addition, G. mellonella has been reported has as a model for several intracellular bacteria, such as Burkholderia pseudomallei, Campylobacter jejuni, Francisella tularensis, and Coxiella burnetti (Aperis et al., 2007; Schell et al., 2008; Champion et al., 2010; Wand et al., 2011) which are closely related to L. pneumophila. For these reasons, alternative models of infection using insects are increasingly used to characterize the virulence of bacterial strains before passing to animal models.

A major component of the larvae's immunity is the presence of an innate immune system with hemocytes, resembling alveolar macrophages in humans, which L. pneumophila is able to infect and replicate making this a suitable model to study pathogenicity (Harding et al., 2012). Furthermore, it was reported that L. pneumophila infection in G. mellonella is dependent of the strain, infectious dose, growth phase and dot/icm presence (Harding et al., 2012, 2013a,b; Aurass et al., 2013; McAdam et al., 
2014), making this model suitable to assess putative differences in virulence between $L$. pneumophila strains.

Here we sought to determine if unrelated L. pneumophila strains, isolated from different environments and with distinct genetic backgrounds (Chien et al., 2004; Costa et al., 2005, 2010, 2012, 2014) exhibited different levels of virulence using G. mellonella infection model. With these data we aimed to elucidate if there is a subset of L. pneumophila isolates specially adapted to produce disease and if this feature could be related with their environmental origin. Our results showed that all tested strains caused mortality in G. mellonella, in a dosedependent and strain-specific mode. Nevertheless no link could be established between L. pneumophila virulence, environmental origin, and sequence-based typing profile. These data strongly suggest that L. pneumophila infection is linked with the ability of some strains to thrive and persist in man-made environments as a result of environmental selection, rather than with their ability to cause disease.

\section{MATERIALS AND METHODS}

\section{Legionella pneumophila Strains and Galleria mellonella Larvae}

L. pneumophila strains were selected from several environments in order to capture the maximum genetic variability determined in early studies from $d o t / i \mathrm{~cm}$, sidJ and T2S related genes (Costa et al., 2010, 2012, 2014). These included 7 isolates from natural environments, 5 isolates from man-made environments, and 4 clinical-related L. pneumophila strains (Table 1). From those, 12 belonged to the L. pneumophila subsp. pneumophila, two to the L. pneumophila subsp. fraseri and the remaining two strains to the L. pneumophila subsp. pascullei. G. mellonella larvae were obtained from the Institute for Bioengineering and Biosciences, IST, Lisbon, Portugal and stored at room temperature in the dark.

\section{Virulence Determination in Galleria mellonella}

L. pneumophila strains were cultured on charcoal-yeast extract (CYE) plates at $37^{\circ} \mathrm{C}$ for 4 days prior to inoculation into ACES [N-(2-acetamido)-2-aminoethanesulfonic acid]-yeast extract (AYE). For each $L$. pneumophila strain the growth curves were determined in AYE at optical density at $600 \mathrm{~nm}$ $\left(\mathrm{OD}_{600}\right)$ to assess the corresponding growth rates in order to confirm that all strains were in post-exponential phase at $21 \mathrm{~h}$, as required for the infection protocol. Additionally, determining L. pneumophila CFU/ml values corresponding to an $\mathrm{OD}_{00}$ of 1 allowed to prepare solutions with pre-determined concentrations to be tested (injected) in G. mellonella (Table S1). Briefly, for each L. pneumophila strain one full loop of bacteria from BCYE plates was suspended in $3 \mathrm{ml}$ of AYE and incubated for 18-19 hours at $37^{\circ} \mathrm{C}$ with $200 \mathrm{rpm}$ in a shaking incubator. A tube with AYE was used as control to ensure sterility of the media. After incubation, $\mathrm{OD}_{600}$ was measured and new inoculums were prepared in $3 \mathrm{ml}$ of $\mathrm{AYE}$ to a final $\mathrm{OD}_{600}$ of 0.1 . Tubes were left in a shaking incubator as described above, this time for $21 \mathrm{~h}$. After this incubation time, $\mathrm{OD}_{600}$ was measured again and the inoculum enumerated using the Drop Plate Method to determine the relation between $\mathrm{OD}_{600}$ and $\mathrm{CFU} / \mathrm{ml}$. According to the predetermined relation between $\mathrm{OD}_{600}$ and $\mathrm{CFU} / \mathrm{ml}$ the injection concentration was normalized for each tested strain.

The infection of G. mellonella was performed as previously described (Harding et al., 2012). Briefly, after $21 \mathrm{~h}$ of growth, bacteria were diluted in Dulbecco's phosphate-buffered saline (PBS) to $\mathrm{OD}_{600}$ corresponding to the required $\mathrm{CFU} / \mathrm{ml}$. To be suitable for injection, larvae were at the 5 th or 6th instars stage which corresponded approximately to $2-3 \mathrm{~cm}$ in length and had no sign of darkening. The injection site (the hindmost left proleg) was disinfected with ethanol and the injection was performed using a micrometer adapted to control the injection volume onto a micro-syringe (Mil-Homens et al., 2010). G. mellonella larvae were injected with $10 \mu \mathrm{l}$ of bacterial suspension prepared and incubated at $37^{\circ} \mathrm{C}$ in the dark. Ten larvae were injected for each strain of L. pneumophila tested. As a control, 10 larvae were injected with D-PBS alone, and 10 untreated insects were included in every experiment. Assays were allowed to proceed for $72 \mathrm{~h}$ as pupa formation could occasionally be seen after that (Harding et al., 2012). At least three independent replicates of each experiment were performed. To confirm the injected $\mathrm{CFU} / \mathrm{ml}$ of L. pneumophila, the injected solutions were plated in BCYE plates and enumerated using the Drop Plate Method.

For each L. pneumophila strain the injected-larvae were individually examined after $18,24,48$ and $72 \mathrm{~h}$ post-injection (p.i.) for time of death and for several phenotypic characteristics that correlate with different stages of disease (Tsai et al., 2016). Namely, melanization (black, black spots on brown larvae, spots on beige larvae and no melanization), cocoon formation (full cocoon and no cocoon) and movement (move without stimulation, move when stimulated and no movement). The G. mellonella Health Index Scoring System (Loh et al., 2013) was used to assesses the larvae health status by assigning scores according to 4 major observations: larvae mobility, cocoon formation, melanization, and survival.

\section{Statistical Analysis}

All quantitative data were obtained from at least three independent assays. Standard deviation was used to calculate error bars. The Mantel-Cox test to determine $p$-values for survival and the ordinary one-way ANOVA of Unpaired $t$-test data to determine $p$-values for health index were performed using GraphPad Prism 7.01 software. Differences were considered to be statistically significant if the $p$-value was lower than 0.05 .

\section{L. pneumophila Allelic Profiling}

Genotyping was performed according to the 7-gene protocol from the European Working Group for Legionella Infections (EWGLI) SBT scheme (http://www.hpa-bioinformatics.org.uk/ legionella/legionella_sbt/php/sbt_homepage.php). The seven gene fragments flaA, pilE, asd, mip, mompS, proA and neuA were amplified and sequenced as previously described (Gaia et al., 2005; Ratzow et al., 2007; Mentasti et al., 2014). Designation of alleles was performed according to the EWGLI SBT database and its combination was represented as an ordered numerical vector. Strain data is summarized in Table 2. The partial sequences of 
TABLE 1 | Characteristics of $L$. pneumophila strains used in this study.

\begin{tabular}{|c|c|c|c|c|c|}
\hline Strain designation & Source & Country & Subspecies & Serogroup & Isolation references \\
\hline HRD2 & Clinical & Portugal (Central) & L. pneumophila subsp. pneumophila & 1 & Costa et al., 2010 \\
\hline Lansing3 (ATCC 35251) & Clinical & USA & L. pneumophila subsp. fraseri & 15 & Brenner et al., 1988 \\
\hline Los Angeles1 (ATCC 33156T) & Clinical & USA & L. pneumophila subsp. fraseri & 4 & McKinney et al., 1979 \\
\hline Philadelphia1 (ATCC 33152T) & Clinical & USA & L. pneumophila subsp. pneumophila & 1 & Chien et al., 2004 \\
\hline HUC1 & Man-made & Portugal (Central) & L. pneumophila subsp. pneumophila & 1 & Costa et al., 2010 \\
\hline IMC23 & Man-made & Portugal (Central) & L. pneumophila subsp. pneumophila & 1 & Veríssimo et al., 1990 \\
\hline MICU B (ATCC 33735) & Man-made & UK & L. pneumophila subsp. pascullei & 5 & Brenner et al., 1988 \\
\hline Por3 & Man-made & Portugal (Southern) & L. pneumophila subsp. pneumophila & 1 & Costa et al., 2010 \\
\hline U8W (ATCC 33737T) & Man-made & UK & L. pneumophila subsp. pascullei & 5 & Brenner et al., 1988 \\
\hline Ice27 & Natural & Iceland & L. pneumophila subsp. pneumophila & 1 & Costa et al., 2010 \\
\hline Aço12 & Natural & Portugal (Azores) & L. pneumophila subsp. pneumophila & 6 & Veríssimo et al., 1991 \\
\hline AçO22 & Natural & Portugal (Azores) & L. pneumophila subsp. pneumophila & 3 & Veríssimo et al., 1991 \\
\hline Aço5 & Natural & Portugal (Azores) & L. pneumophila subsp. pneumophila & 6 & Veríssimo et al., 1991 \\
\hline Agn2 & Natural & Italy (Agano) & L. pneumophila subsp. pneumophila & 1 & Costa et al., 2010 \\
\hline Ger10 & Natural & Portugal (Northern) & L. pneumophila subsp. pneumophila & 1 & Costa et al., 2010 \\
\hline NMex1 & Natural & USA (New Mexico) & L. pneumophila subsp. pneumophila & 6 & Marrão et al., 1993 \\
\hline
\end{tabular}

L. pneumophila flaA, pile, asd, mip, mompS, proA and neuA genes determined in this study were deposited in GenBank under the accession numbers MG979220 to MG979296.

\section{Minimum Spanning Tree Based on Allelic Profiles of $L$. pneumophila STs}

The EWGLI SBT database (http://www.ewgli.org/) included data from 11927 isolates (with Sequence Type - ST, attributed), from 63 countries, representing 2467 distinct STs, at the time this analysis was conducted (17.10.2017). The eBURST algorithm (Francisco et al., 2009) implemented in the PHYLOViZ2.0 software (Nascimento et al., 2017) was used to construct a full minimum spanning tree (MST) displaying the relationships between the allelic profile of the studied strains and other STs described worldwide since groups are all believed to be descended from the same founding genotype (the primary founder) (Correia et al., 2016). STs that couldn't be assigned to any group were called singletons. The statistical confidences for the founders were assessed using 1000 bootstrap re-samplings. Clustering of ST profiles from the studied strains was determined by calculating the Hamming distance using the Unweighted Pair Group Method with Arithmetic Averages (UPGMA), implemented in the PHYLOViZ2.0 software (Nascimento et al., 2017).

\section{STs Clustering and Phylogenetic Analysis}

The similarity between the ST profiles was determined by calculating the Pearson's coefficient with correction: $d=(1-r)$ $\mathrm{x}$ 100. Allelic profile were clustered with the Unweighted Pair Group Method with Arithmetic Averages (UPGMA), employing the DendroUPGMA computer program (http://genomes.urv. cat/UPGMA/) (Garcia-Vallvé and Palau, 1999). Phylogenetic analyses were performed using MEGA5 package (Tamura et al., 2011). Alignment against the corresponding genes found in the EWGLI SBT database for the L. pneumophila strains included in this analysis (Table 1), was performed using the multiple alignment CLUSTAL software (Higgins, 1994), included on MEGA5 package. Maximum likelihood phylogenetic tree was obtained for the concatenated alignment with PhyML 3.0 (Guindon and Gascuel, 2003), using the most appropriate model of nucleotide substitution and likelihood scores assessed by TOPALi V2.5 (Milne et al., 2004) and by jModeltest (Posada, 2008). The best model was determined by using the Akaike Information Criterion (AIC) (Akaike, 1974; Posada and Buckley, 2004). Supports for the nodes were evaluated by bootstrapping with 1000 pseudoreplicates.

\section{RESULTS}

\section{Mortality of G. mellonella Infected With Diverse Isolates of $L$. pneumophila Is Dose Dependent}

Determining L. pneumophila $\mathrm{CFU} / \mathrm{ml}$ corresponding to an $\mathrm{OD}_{600}$ of 1 for each of the tested strains was mandatory to prepare suspensions for injection with a pre-determined concentration in order to standardize the experiment. Differences between the $\mathrm{CFU} / \mathrm{ml}$ values that corresponded to an $\mathrm{OD}_{600}$ of 1 were observed between strains. The most notorious difference was observed for strain Por3 with a CFU/ml value of $2.6 \times 10^{8}$, one order of magnitude lower than the one recorded of all other strains (Table S1). These results were taken into account in order to guarantee that the concentration of injected bacteria was the same for all L. pneumophila strains.

As previously mentioned, one of the goals of this study was to determine if there were differences between L. pneumophila strains regarding their capability to induce larvae mortality. To answer this question, three different injection concentrations $\left(10^{5}, 10^{6}\right.$ and $10^{7} \mathrm{CFU} /$ larvae $)$ were tested in G. mellonella in three L. pneumophila strains. This allowed us to select the 
TABLE 2 | Characteristics of L. pneumophila strains used in this study.

\begin{tabular}{|c|c|c|c|c|c|c|c|c|c|c|c|}
\hline \multicolumn{3}{|c|}{ Isolates used in this study } & \multicolumn{9}{|c|}{ L. pneumophila isolates present in the ESGLI SBT database } \\
\hline \multirow[t]{2}{*}{ Strain designation } & \multirow[t]{2}{*}{ Source } & \multirow[t]{2}{*}{$\mathbf{S T}^{\mathbf{a}}$} & \multirow[t]{2}{*}{$N^{b}$} & \multicolumn{4}{|c|}{ Source (\%) } & \multicolumn{2}{|c|}{ Origin } & \multicolumn{2}{|c|}{ Locus variant } \\
\hline & & & & Man made & Clinical & Natural & Unknown & Country & Continent ${ }^{\mathrm{c}}$ & SLVd & DLVe \\
\hline HRD2 $2^{\#}$ & Clinical & 44 & 61 & 9.8 & 88.5 & 1.6 & - & 9 & Am, $E$ & 4 & 18 \\
\hline Lansing3 (ATCC 35251) & Clinical & 336 & 2 & - & 100 & - & - & 2 & Am, As & 6 & 8 \\
\hline Los Angeles1 (ATCC 33156') & Clinical & 1,334 & 6 & 50 & 50 & - & - & 4 & Am, As, E & 7 & 9 \\
\hline Philadelphia1 (ATCC $33152^{\top}$ ) & Clinical & 36 & 61 & 8.2 & 91.8 & - & - & 11 & Am, As, E & 18 & 43 \\
\hline IMC23 & Man-made & $2,370^{\star}$ & 1 & - & 100 & - & - & 1 & $E$ & 0 & 0 \\
\hline $\mathrm{HUC1}^{\#}$ & Man-made & $2,353^{*}$ & 1 & 100 & - & - & - & 1 & $E$ & 3 & 23 \\
\hline MICU B (ATCC 33735) \# & Man-made & 1,335 & 4 & 100 & - & - & - & 2 & $E$ & 0 & 1 \\
\hline Por3 & Man-made & 1 & 1486 & 43 & 56 & - & 0.47 & 32 & Am, As, E, & 62 & 63 \\
\hline U8W (ATCC $33737^{\top}$ ) & Man-made & 1,335 & 4 & 100 & - & - & - & 2 & $E$ & 0 & 1 \\
\hline Ice27 & Natural & $2,334^{*}$ & 1 & - & - & 100 & - & 1 & $E$ & 0 & 0 \\
\hline Aço12 & Natural & 68 & 64 & 56 & 40 & 1.6 & 1.6 & 13 & Am, As, E & 19 & 49 \\
\hline AçO22 & Natural & $2,333^{\star}$ & 1 & - & - & 100 & - & 1 & $E$ & 0 & 4 \\
\hline Aço5 & Natural & $2,354^{\star}$ & 1 & - & - & 100 & - & 1 & $E$ & 6 & 28 \\
\hline Agn2 $\#$ & Natural & $2,336^{\star}$ & 1 & - & - & 100 & - & 1 & $E$ & 1 & 2 \\
\hline Ger10\# & Natural & 1,362 & 10 & 60 & 30 & 10 & - & 5 & $E$ & 18 & 93 \\
\hline NMex1 $\#$ & Natural & 1,892 & 2 & 50 & - & 50 & - & 2 & Am, As & 6 & 30 \\
\hline
\end{tabular}

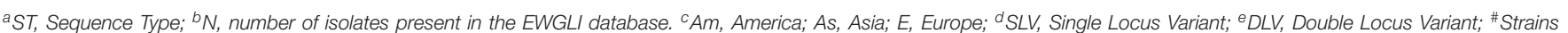
significantly different from L. pneumophila Philadelphia 1 type strain on their ability to induce higher G. mellonella mortality; ${ }^{*} S T$ reported in this study.

injection concentration that showed greater differences on larvae survival to be used in the following experiments.

From the tested conditions, no mortality was observed for the $10^{5} \mathrm{CFU} /$ larvae injection concentration within the experimental time course, similar to what was observed for the D-PBS injected larvae, serving as control (Figure S1a).

Differences on larvae survival between strains were observed after injection with $10^{6} \mathrm{CFU} /$ larvae. In the first $24 \mathrm{~h}$ p.i., $100 \%$ of larvae survival was registered for all strains. The differences between strains were more pronounced with time. Namely, for IMC23 and Philadelphia1 a 20\% larvae mortality occurred at $48 \mathrm{~h}$ p.i., while for strain Aço5 this value was $10 \%$. In addition, Philadelphia1-infected G. mellonella had a larvae mortality of $60 \%$ after $72 \mathrm{~h}$ p.i. while for strains IMC23 and Aço5 the mortality values were of $80 \%$. Nevertheless, we did not observe $100 \%$ of mortality on larvae injected with this concentration. Similarly, no mortality was observed for the D-PBS injected larvae serving as control (Figure S1b).

At the highest concentration no major differences on larvae survival were observed between strains since all induced a larvae mortality of 70,90 , and $100 \%$ at 24,48 and $72 \mathrm{~h}$ p.i., respectively. Nevertheless, the survival curve differed between strains at $18 \mathrm{~h}$ p.i., where strain Philadelphia 1 induced a larvae mortality of $20 \%$ while strain Aço5 and IMC23 induced $40 \%$ of larvae mortality. No mortality was observed for the D-PBS injected larvae serving as control (Figure S1c).

Ultimately, differences in larvae survival were observed between the three injection concentrations since G. mellonella was able to survive low infectious dose but succumbed to higher doses. These observations confirmed that L. pneumophila virulence in G. mellonella was dose dependent (Harding et al., 2012). As infection with $10^{7} \mathrm{CFU} /$ larvae caused very rapid death, any differences in virulence of the tested strains would be disguised. Hence, in the following experiments, we injected larvae with an infectious dose of $10^{6} \mathrm{CFU}$ in order to identify differences on virulence between strains.

\section{L. pneumophila Virulence in G. mellonella Was Strain and Time Dependent}

Prior work evidenced that three usually studied SG1 L. pneumophila strains caused death of at least, $70 \%$ of G. mellonella larvae accompanied by severe organ damage translated in larvae melanization. These results were dependent of the strain, infectious dose, growth phase and presence of T4SS (Harding et al., 2012). We intended to corroborate those evidences and broader the overall picture by using a wider group of unrelated L. pneumophila strains, isolated from different environments and with distinct genetic backgrounds (Chien et al., 2004; Costa et al., 2005, 2010, 2012, 2014) (Table 1). Briefly, to determine the intrinsic capability of L. pneumophila strains to induce mortality in G. mellonella, 10 larvae were injected with $10^{6} \mathrm{CFU} /$ larvae of each tested strain and their survival was monitored for $72 \mathrm{~h}$ (Table S2). A control group was also tested by injection with $10 \mu \mathrm{l}$ of D-PBS.

Foremost, L. pneumophila strains demonstrated significantly $(P<0.0001)$ differences on the capability to induce $G$. mellonella mortality (Figure 1A). Indeed, some strains induced 100\% larvae mortality while others only induced some mortality, although at distinct rates (Figure 1A). Namely, strain MICU B induced 


A
\begin{tabular}{|l|l|c|c|c|c|}
\hline \multirow{2}{*}{ L. pneumophila strains } & \multirow{2}{*}{ Source } & \multicolumn{4}{|c|}{ Hours post-infection } \\
\cline { 3 - 6 } & & 18 & 24 & 48 & 72 \\
\hline Aço12 & Natural & $100 \pm 0.0$ & $100 \pm 0.0$ & $80 \pm 8.1$ & $70 \pm 8.1$ \\
\hline Los Angeles 1 & Clinical & $80 \pm 5.8$ & $80 \pm 0.0$ & $80 \pm 0.0$ & $60 \pm 5.8$ \\
\hline Por3 & Man-made & $90 \pm 5.8$ & $80 \pm 11$ & $60 \pm 5.8$ & $50 \pm 5.8$ \\
\hline Ice27 & Natural & $80 \pm 0.0$ & $80 \pm 0.0$ & $60 \pm 5.8$ & $50 \pm 5.8$ \\
\hline Philadelphia 1 & Clinical & $100 \pm 0.0$ & 1000.0 & $70 \pm 10$ & $40 \pm 10$ \\
\hline Lansing 3 & Clinical & $60 \pm 0.0$ & $60 \pm 5.8$ & $40 \pm 5.8$ & $40 \pm 5.8$ \\
\hline Aço22 & Natural & $100 \pm 0.0$ & $100 \pm 0.0$ & $70 \pm 4.7$ & $30 \pm 5.8$ \\
\hline Aço5 & Natural & $100 \pm 0.0$ & $100 \pm 0.0$ & $90 \pm 13$ & $20 \pm 7.0$ \\
\hline IMC23 & Man-made & $100 \pm 0.0$ & $100 \pm 0.0$ & $80 \pm 8.1$ & $20 \pm 7.0$ \\
\hline U8W & Man-made & $90 \pm 11$ & $90 \pm 11$ & $60 \pm 15$ & $10 \pm 11$ \\
\hline Ger10 & Natural & $80 \pm 5.8^{*}$ & $50 \pm 8.1^{*}$ & $40 \pm 5.8^{*}$ & $0 \pm 0.0^{*}$ \\
\hline HUC1 & Man-made & $70 \pm 5.8^{* * *}$ & $40 \pm 5.8^{* * *}$ & $0 \pm 5.8^{* * * *}$ & $0 \pm 0.0^{* * *}$ \\
\hline Agn2 & Natural & $50 \pm 10^{* * * *}$ & $20 \pm 5.8^{* * * * *}$ & $0 \pm 5.8^{* * * * *}$ & $0 \pm 0.0^{* * * * *}$ \\
\hline Nmex1 & Natural & $100 \pm 0.0^{* * * *}$ & $0^{* * * *}$ & $0^{* * * * *}$ & $0^{* * * *}$ \\
\hline HRD2 & Clinical & $100 \pm 0.0^{* * * *}$ & $0^{* * * *}$ & $0^{* * * *}$ & $0^{* * * *}$ \\
\hline MICU B & Man-made & $0^{* * * *}$ & $0^{* * * *}$ & $0^{* * * * *}$ & $0^{* * * * *}$ \\
\hline
\end{tabular}

Larval survival (\%)

\begin{tabular}{|lllllllllll|}
\hline 100 & 90 & 80 & 70 & 60 & 50 & 40 & 30 & 20 & 10 & 0 \\
\hline
\end{tabular}

\section{B}

\begin{tabular}{|l|l|c|c|c|c|}
\hline \multirow{2}{*}{ L. pneumophila strains } & \multirow{2}{*}{ Source } & \multicolumn{4}{|c|}{ Hours post-infection } \\
\cline { 3 - 6 } & & 18 & 24 & 48 & 72 \\
\hline Aço12 & Natural & 9,7 & 8,6 & 5,4 & 5,6 \\
\hline Los Angeles 1 & Clinical & 6,4 & 6,2 & 5,1 & 2,8 \\
\hline Por3 & Man-made & 6,8 & 4,4 & 3,1 & 3,3 \\
\hline Ice27 & Natural & 7,7 & 6,3 & 3,6 & 2,8 \\
\hline Philadelphia 1 & Clinical & 9,4 & 7,9 & 4,1 & 1,6 \\
\hline Lansing 3 & Clinical & 4,6 & 3,7 & 2,7 & 1,7 \\
\hline Aço22 & Natural & 9,4 & 8,9 & 5,2 & 1,2 \\
\hline Aç05 & Natural & 9,4 & 8,6 & 5,0 & 0,8 \\
\hline IMC23 & Man-made & 8,5 & 7,9 & 4,0 & 0,7 \\
\hline U8W & Man-made & 7,2 & 6,3 & 2,7 & 0,1 \\
\hline Ger10 & Natural & 5,0 & 2,9 & 1,7 & 0,0 \\
\hline HUC1 & Man-made & 4,1 & 1,7 & 0,1 & 0,0 \\
\hline Agn2 & Natural & 2,1 & 0,9 & 0,1 & 0,0 \\
\hline Nmex1 & Natural & 6,5 & 0,0 & 0,0 & 0,0 \\
\hline HRD2 & Clinical & 6,5 & 0,0 & 0,0 & 0,0 \\
\hline MICU B & Man-made & 0,0 & 0,0 & 0,0 & 0,0 \\
\hline
\end{tabular}

Health Index

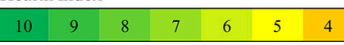

FIGURE 1 | L. pneumophila virulence in G. mellonella is strain-specific. (A) Larvae survival (\%) (B) Health Index. G. mellonella larvae were infected with $10^{6} \mathrm{CFU}$ of each of the tested strains or D-PBS, and the survival rate and health status was monitored for $18,24,48$, and $72 \mathrm{~h}$. Injection with D-PBS was used as control and no mortality was observed (data not shown). L. pneumophila-induced mortality in G. mellonella was dependent on the tested strain with significant differences $(P<0.0001)$ between strains. In addition for the larvae survival, each strain was also statistically compared with L. pneumophila Philadelphia 1 and differences were considered significant if ${ }^{\star} P<0.05$, highly significant if ${ }^{\star \star \star} P<0.001$ and extremely significant if ${ }^{* \star \star \star} P<0.0001$. Results are the mean of at least three independent experiments; \pm standard deviation.

$100 \%$ larval mortality after $18 \mathrm{~h}$ p.i., while NMex1 and HRD2 showed the same results after $24 \mathrm{~h}$ p.i. Agn2 and HUC1 strains also induced $100 \%$ larval mortality but in this case after $48 \mathrm{~h}$ p.i. Lastly, Ger10 hit $100 \%$ mortality after $72 \mathrm{~h}$ p.i. In contrast, some larvae survival was seen for the remaining strains despite relevant differences on the infection kinetics (\% induced larvae mortality); namely for strains U8W (90\%), IMC23 and Aço5 (80\%), Aço22 (70\%), Philadelphia 1 and Lansing3 (60\%), Ice27 (50\%) and Los Angeles1 (40\%) (Figure 1A). Nevertheless they all had a common characteristic, all tested strains induced death at a given point.

These results allow us to conclude that larvae mortality kinetics and infection profile were strain specific. Moreover, a panoply of virulence-related phenotypes were observed as some of the tested strains were defective in their ability to cause disease; while others were highly pathogenic. Since a correlation has been established between the virulence variation in G. mellonella model and the virulence variation observed in mammalian cell culture and animal models for several human pathogens (Ramarao et al., 2012; Cook and McArthur, 2013; Champion et al., 2016; Tsai et al., 2016; Velikova et al., 2016; Barnoy et al., 2017) altogether our data suggest the existence of potential differences on the ability of $L$. pneumophila strains to induce disease in humans.

\section{All L. pneumophila Strains Were Pathogenic in G. mellonella}

In order to detect more subtle differences between L. pneumophila strains ability to cause disease, the G. mellonella Health Index Scoring System was used (Loh et al., 2013; Tsai et al., 2016). Briefly, each larvae was simultaneously scored for the survival rate (Figure 1A) and health status (Figure 1B), through the observation of several features; namely activity, cocoon formation and melanization, that correlates with different stages of disease. As previously described, higher activity and cocoon formation correspond to a healthier larvae. Melanization refers to the synthesis and deposition of melanin that occurs as a result of an immune response against infection, as melanin aids to encapsulate and kill pathogens. This process usually starts with distinctive black spots that increase in size followed by the complete melanization (black larvae) that correlates with death of the larvae soon after (Loh et al., 2013). A score was assigned to each observation and an overall health index score was calculated for each larvae, ranging from 10 (healthy larvae) to 0 (dead larvae) (Tsai et al., 2016) (Figure 1B and Table S2).

Decreasing health index scores relates well with increasing virulence of L. pneumophila strains (Figure 1), and it was clear that all strains induced morphologic alterations in the infected larvae at a certain point. Indeed, larvae injected with the most virulent strains showed a rapid decline in health that matched the survival rate. Remarkably, even in strains that induced partial larvae mortality, the survivors displayed reduced health index values (Figure 1B). This reinforced that all tested strains were pathogenic in G. mellonella.

Nevertheless, despite strains Aço12 and Por3 induced respectively $30 \%$ and $50 \%$ larval mortality at the end of the experiment, the survivor's showed signs of recovery between 48 and $72 \mathrm{~h}$ p.i. based on the health index (Table S2). Observing the data from morphological characteristics regarding these strains, it was noticed that until $48 \mathrm{~h}$ p.i. larvae presented common alterations, such as the increase of darkening, no 
cocoon formation and slow movement. Nevertheless, between 48 and $72 \mathrm{~h}$ p.i., larvae showed signs of recovery. Indeed, the number of dark larvae stabilized or even decreased with a concomitant increase in the number of normal color larvae. The same happened for cocoon formation and movement. These observations suggested that some larvae could partially overcome infection with L. pneumophila strains Aço12 and Por3 (Figure 1B). Since L. pneumophila strains used in this work had distinct virulence-related genetic repertoire (Costa et al., 2010, 2012, 2014), one possible explanation for the abovementioned results could be related with these strains genetic context translated in a limited ability to replicate or persist in the larvae.

\section{The Origin of $L$. pneumophila Strains Does Not Correlate With Virulence in G. mellonella}

As previously mentioned, studies supported the premise that only a specific subset of environmental L. pneumophila strains were actually capable of producing infection in humans (Coscolla and Gonzalez-Candelas, 2009; David et al., 2016). In line, our above-mentioned results corroborate that L. pneumophila strains demonstrated statistically significant $(P<0.0001)$ differences on their ability to induce G. mellonella mortality (Figure 1). To further investigate this hypothesis statistical analysis was performed to determine if the pathogenicity of L. pneumophila in G. mellonella was related with the origin of the strains since nothing is known about the nature and extent of virulence variation in isolates from natural environments. This is the first report in which natural environmental L. pneumophila strains were tested for their ability to cause infection.

Regarding strains isolated from natural environments, significant differences $(P<0.0001)$ were observed on their ability to kill larvae. For instance, strain NMex1 induced $100 \%$ of larvae mortality $24 \mathrm{~h}$ p.i. while strain Aço12 only induced $30 \%$ of larvae mortality and in a longer period of time (72 h p.i.) (Figure 1A). Similarly, significant differences $(P<0.0002)$ were observed on the ability of man-made environmental strains to kill larvae. Namely, strains MICU B and HUC1 were capable of inducing $100 \%$ of larvae mortality, while Por3 only induced $50 \%$ of larvae mortality within the experiment time frame (Figure 1B). Regarding the clinical-related strains, a $P<0.0001$ supported significant differences among strains, as previously observed for other clinical strains (Harding et al., 2012). Namely, HRD2 induced $100 \%$ of larvae mortality at $24 \mathrm{~h}$ p.i., while Los Angeles 1 was only capable of causing $40 \%$ of larvae mortality at $72 \mathrm{~h}$ p.i. (Figure 1A). Decreasing health index scores relates well with increasing virulence of L. pneumophila strains (Figure 1), and it was clear that all strains induced morphologic alterations in the infected larvae at a given point.

Our results show that L. pneumophila pathogenicity is not related with the origin of the strains. In fact, we were able to identify strains capable of inducing $100 \%$ of mortality in G. mellonella isolated from all environmental types. Likewise, strains with a reduced ability to cause death were also isolated from all environmental types (Figure 1).
The genus Legionella comprises over 60 species, being L. pneumophila the leading cause of Legionnaire's Disease (Khodr et al., 2016). Based on DNA-DNA hybridization tests L. pneumophila was classified into three subspecies, L. pneumophila subsp. pneumophila, L. pneumophila subsp. fraseri and L. pneumophila subsp. pascullei (Brenner et al., 1988). L. pneumophila subsp. pneumophila has been associated with almost $85-90 \%$ of the disease cases worldwide (David et al., 2016). Nevertheless, it has not been possible to establish a correlation between this incidence and their environmental persistence or virulence (Doleans et al., 2004; Harrison et al., 2007; Kozak et al., 2009; David et al., 2016). In order to determine if the aforementioned predominance of L. pneumophila subsp. pneumophila in disease cases could be related with higher virulence, the pathogenicity of the three L. pneumophila subsp. was analyzed. All subsp. caused time-dependent death of at least $40 \%$ of the G. mellonella larvae; in fact L. pneumophila subsp. pascullei strain MICU B caused significantly $(P<0.005)$ higher mortality than L. pneumophila subsp. pneumophila strains, $18 \mathrm{~h}$ p.i. Considering the link previously determined between the virulence of several human pathogens in G. mellonella and their ability to cause disease (Cook and McArthur, 2013; Tsai et al., 2016), these results suggest that some L. pneumophila strains are more frequently related with human disease, not because they are more virulent, but most probably because of their wider environmental distribution, higher concentration or ability to persistence in the man-made environments.

\section{Comparison Between the Ability of L. pneumophila Type Strain Philadelphia 1 and the Other Tested Strains to Infect G. mellonella}

Philadelphia 1 is the type strain of $L$. pneumophila subsp. pneumophila (SG1) and is derived from the original clinical isolate collected during the first recognized outbreak of Legionnaires' disease (Fraser et al., 1977; McDade et al., 1977). This strain has been used in numerous laboratories worldwide and was the basis of the research conducted on this important human pathogen (Chien et al., 2004; Gomez-Valero and Buchrieser, 2013; Cunha et al., 2016). Since the capability of Philadelphia 1 to infect $G$. mellonella has already been tested by others (Harding et al., 2012), we decided to use it as a reference strain for comparison purposes. This strategy allowed us to ascertain if there were, or not, significant differences on the ability to kill larvae between strains recovered from other environments, namely natural and man-made environments, in opposition to clinical-related strains.

Indeed, significant differences were observed on the ability of L. pneumophila strains to induce larvae mortality when compared to Philadelphia 1 (Figure 1). Surprisingly, the aforementioned strains included natural environmental isolates, namely Agn2 and NMex1 $(P<0.0001)$ and Ger10 $(P<0.05)$, and as expected clinical-related and man-made environmental isolates, namely HRD2 and MICU B $(P<0.0001)$ and HUC1 $(P<0.001)$. All other strains exhibited a behavior that was not significantly different from the reference strain (Figure 1). 
Since Philadelphia 1 has been responsible for several outbreaks of Legionnaire's Disease (Muder and Yu, 2002; Beauté et al., 2013; David et al., 2016; Beauté and Network on behalf of the E. L. D. S., 2017), the identification of strains with the same capability to induce larvae mortality may suggests that they could have identical potential to cause disease in humans. Moreover, those strains were isolated from both natural and man-made environments, contradicting the hypothesis that only a specific subset of environmental isolates is actually capable of producing infection in humans (Coscolla and Gonzalez-Candelas, 2009; Harrison et al., 2009; David et al., 2016). Our data reinforces that the occurrence of human infection may not be related with the increased capability of some strains to induce disease. In fact, the decisive factor is probably linked with Legionella ability to persist and multiply to high numbers in man-made environments that generate contaminated aerosols that might be dispersed over a wide area.

\section{Relation Between the Studied Strains and the Worldwide L. pneumophila Genetic Diversity Inferred From SBT}

An internationally recognized seven-gene molecular typing scheme-Sequence-Based Typing (SBT), has been developed for genotyping L. pneumophila isolates (Gaia et al., 2005; Ratzow et al., 2007) and a curated database has been established by The European Working Group for Legionella Infections (EWGLI). The SBT analysis was performed to ascertain whether there was a correlation between L. pneumophila Sequence Types (STs), source and virulence. Data from reference and type strains was retrieved from the EWGLI SBT database and compared with the STs obtained for the strains used in this study. The results showed that the 16 analyzed strains matched 15 STs. In total, 10 strains clustered in STs previously reported in the EWGLI SBT database, namely Por3 (ST1), Philadelphia1 (ST36), HRD2 (ST44), Aço12 (ST68), Lansing3 (ST336), Los Angeles 1 (ST1334), MICU B and U8W (ST1335), Ger10 (ST1362), and NMex1 (ST1892). The remaining six isolates (37.5\%) displayed newly reported STs, namely Aço22 (ST2333), Ice27 (ST2334), Agn2 (ST2336), HUC1 (ST2353), Aço5 (ST2354), and IMC23 (ST2370) (Table 2). Only L. pneumophila subsp. pascullei strains U8W and MICU B exhibited the same SBT profile.

To establish genotypic relationships and possible correlations with the source of the strains, a cluster analysis at single and double locus variant level was performed by goeBURST algorithm (Figure 2). All strains clustered in a ST group, with the exception of Ice27 (ST2334) and IMC23 (ST2370), which were singletons. Among the 15 STs present in this study, only five had more than 10 isolates in the EWGLI database, namely ST1, ST68, ST44, ST36, and ST1362 (Table 2).

The man-made environmental isolate Por3 matched ST1 (also known as the "Paris-like strain"), detected among 1.468 isolates with a worldwide distribution (32 countries from 5 continents) (Table 2). Recent reports revealed that ST1 belongs to a subset of five STs $(1,23,37,47$, and 62) that are responsible for nearly half of all epidemiologically unrelated cases of Legionnaire's disease (David et al., 2016). Interestingly, from those, only ST1 has been equally isolated from environmental and clinical sources, while the remaining four STs were mostly related with disease (David et al., 2016).

Strain Aço12 was assigned to the widely dispersed ST68 (13 countries from 3 continents) along with 64 strains in the database. Interestingly, no linkage could be established between this ST and the strain source since the isolates have been equally recovered from clinical and environmental sources (Figure 2 and Table 2).

On the other hand, a predominance of clinical isolates (92\%) was clear for the globally disperse ST36, comprised of L. pneumophila type strain Philadelphia 1 and 60 other isolates (11 countries and 3 continents). The same trend was observed for ST44, since more than $88 \%$ of the strains were clinical, regardless its broad distribution (9 countries from 2 continents). The clinical-related strain HRD2 was assigned to this ST along with 60 other strains. Additionally, the natural environmental strain Ger10 belonged to ST1362, comprising predominantly environmental isolates (70\%), recovered from 5 European countries. This is a restrict group with only 10 strains (Figure 2 and Table 2).

Four strains from L. pneumophila subsp. fraseri and pascullei were also included in this analysis, corresponding to three distinct ST profiles. L. pneumophila subsp. fraseri type strain Los Angeles 1 was included in ST1334, along with 6 other isolates from both environmental and clinical sources (50\% each), from 4 different countries in 3 continents. On the other hand, just one other strain matched ST336 along with strain Lansing3, both from clinical samples collected from two continents. The two strains from subsp. pascullei had the same ST1335 profile along with two other environmental isolates from Europe (Figure 2 and Table 2).

These results were in accordance with our initial goal to use unrelated strains. Moreover, the newly reported STs were identified from environmental strains confirming the frequently reported bias on $L$. pneumophila diversity due to the over representation of clinical-related strains (Hyland et al., 2008; Nygård et al., 2008; Sanchez et al., 2008; Phin et al., 2014; Hoisington et al., 2015; van Heijnsbergen et al., 2015, 2016). In addition, several natural environmental strains clustered in clonal complexes along with clinical strains known to cause disease in humans. Since the described natural environmental strains were equally pathogenic in G. mellonella model, one could envision a similar trend in humans. Nevertheless, further studies will be necessary to prove this fate in humans.

\section{Relation Between L. pneumophila ST, Strain Origin and Ability to Induce Mortality in G. mellonella}

In order to capture the relationship between strains inferred from the SBT analysis, an Unweighted Pair Group Method with Arithmetic Averages (UPGMA) was used to build a dendrogram (Figure 3A). The method calculates all the Pearson correlation coefficients between pairs of sets of variables, transforms these coefficients into distances and makes a clustering using the UPGMA algorithm (Garcia-Vallvé and Palau, 


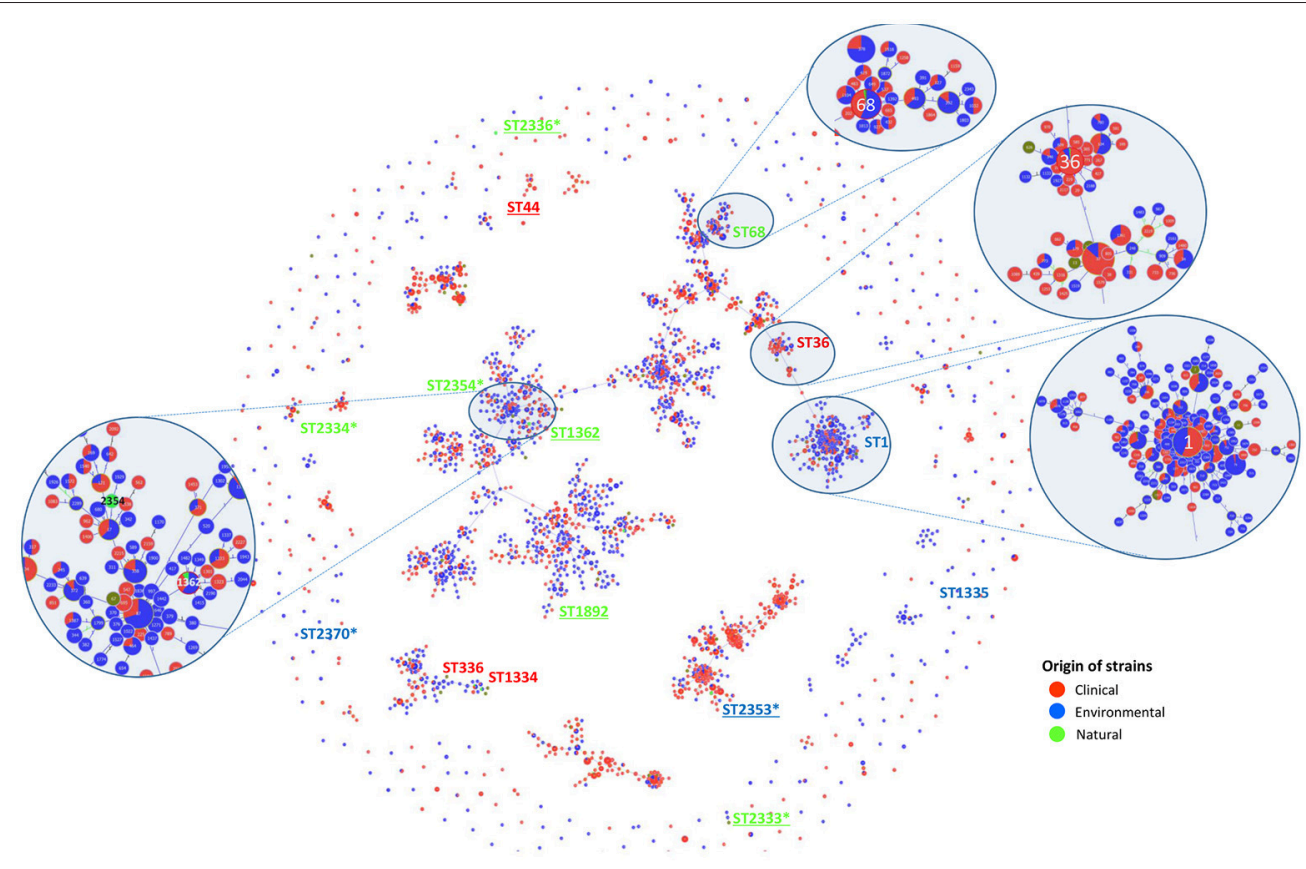

FIGURE 2 | SBT-based representation of the worldwide genetic diversity among L. pneumophila isolates. Relationships between 2,342 allelic profiles of 11,737 isolates (from the EWGLI SBT database) are shown in a goeBURST full minimum spanning tree (MST). The MST connects the ST profiles in a way that the summed distance of all links of the tree is the minimum. STs found in this work are identified. Pies represent the different STs with the size being proportional to haplotype frequencies. The source of the isolates is color coded: clinical-related (red), man-made environment (blue), natural environment (green), and unknown (brown). Underlined strains were significantly different from L. pneumophila Philadelphia 1 type strain on their ability to induce higher G. mellonella mortality; ${ }^{\star}$ New ST described in this study.

1999). These results allow us to conclude that the ST profiles did not reconstruct L. pneumophila species phylogeny, since L. pneumophila strains from the same sub-species were in distinct clusters with strains from other subsp., namely Lansing 3 and Los Angeles 1 (L. pneumophila subsp. fraseri). This was also supported by the phylogeny inferred from the concatenated alignment of the SBT genes since no relation was observed between the distribution of the strains and virulence (Figure 3B). Additionally, no relation could be established between ST's and the origin of strains since isolates from distinct environments were grouped, while strains from the same environmental type were distributed by distinct clusters (Figure 3A).

Previous reports assumed that only a specific subset of L. pneumophila environmental isolates were actually capable of producing infection in humans (Harrison et al., 2007; Coscolla and Gonzalez-Candelas, 2009; David et al., 2016). Our abovementioned results obtained in G. mellonella model did not support this hypothesis since all L. pneumophila strains tested were pathogenic (Ramarao et al., 2012; Cook and McArthur, 2013; Champion et al., 2016; Tsai et al., 2016; Velikova et al., 2016; Barnoy et al., 2017). Nevertheless, our findings raised another hypothesis: could a sub-set of L. pneumophila strains belonging to globally distributed ST's and more often related with clinical cases be more virulent? In order to confirm this hypothesis, we compared the ability of unrelated strains to infect G. mellonella based on their ST (Figure 3A). From our results we were not able to establish a linkage between STs and
L. pneumophila pathogenicity. In fact, the most virulent strains in G. mellonella model (Figure 1) were included in new STs or in STs with very few isolates, with the exception of strain HRD2 (Figure 2 and Table 2). In other words, the STs most commonly associated with clinical cases and widely distributed, namely ST1, ST36, ST336, were represented in this study by strains with an average ability to infect G. mellonella (Figure 3A). Interestingly, the less pathogenic strain assessed in this study, the natural environmental strain Aço12, was clustered in ST68, along with another 64 isolates from man-made environments and clinical cases. Finally, we could not correlate the SBT types, or SBT-based clusters, with the degree of virulence since strains capable of inducing similar results regarding larvae mortality were in separate clusters. Likewise, strains with distinct virulence patterns were clustered (Figure 3A).

The SBT scheme has been used for several years for epidemiological typing of clinical and environmental isolates but we couldn't find any link between pathogenicity and STs. In order to determine if strains belonging to the same ST had the same ability to induce disease we analyzed ST355. This ST comprised two strains of L. pneumophila subsp. pascullei that exhibited significant differences on their pathogenicity since MICU B induced $100 \%$ larvae mortality $18 \mathrm{~h}$ p.i. while U8W did not reach $100 \%$ of larvae mortality during the time frame of the experiment (Figure 1). This indicate that some STs are heterogeneous since they comprise strains with distinct ability to cause disease. In sum, public health strategies based on the SBT scheme analysis 


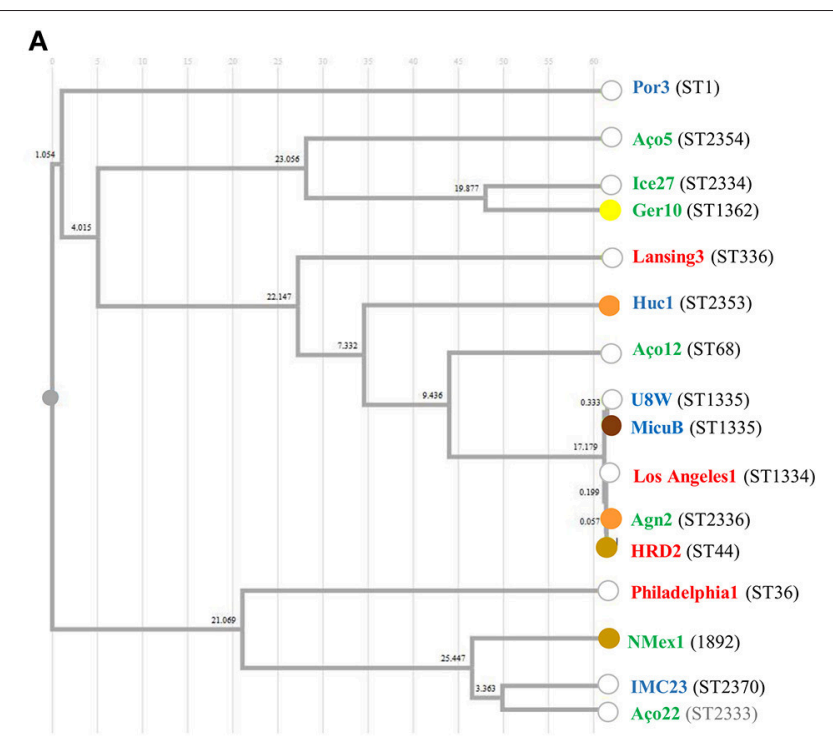

B

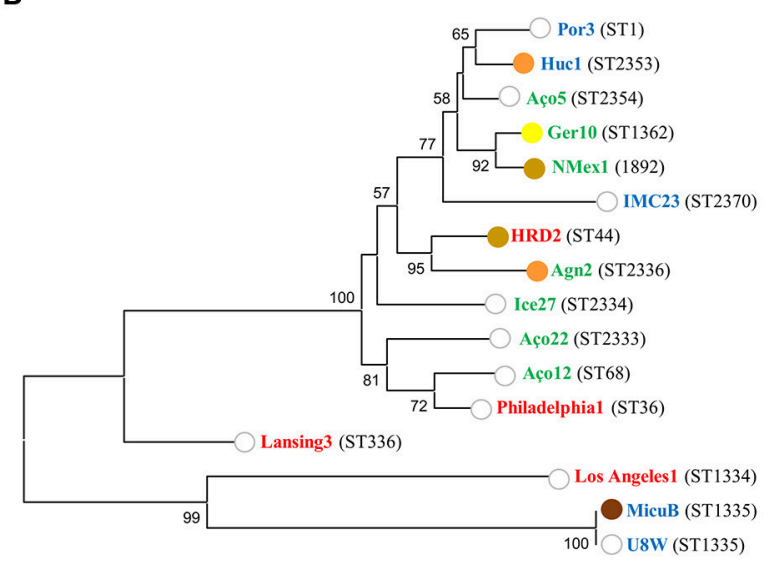

0,0100

FIGURE 3 | Relation between L. pneumophila ST, strain origin and ability to induce mortality in G. mellonella. (A) Cluster analysis dendrogram inferred from the SBT profiles based on the UPGMA algorithm with Pearson correlation coefficient implemented in DendroUPGMA; (B) Maximum likelihood tree from the concatenated alignment of the nucleotide sequences from the SBT analysis. Bootstrap support values (1,000 replicates) for nodes higher than $50 \%$ are indicated. The ability to induce $100 \%$ mortality in G. mellonella over time is color coded: $18 \mathrm{~h}$ (brown); $24 \mathrm{~h}$ (light brown); $48 \mathrm{~h}$ (orange); $72 \mathrm{~h}$ (yellow); do not reach $100 \%$ mortality (white). The source of the isolates is color coded: clinical-related (red), man-made environment (blue), natural environment (green).

should take into account that the major disease-associated clones of L. pneumophila are not associated with higher virulence and that potential variability of virulence-related phenotypes may be found within the same ST.

\section{DISCUSSION}

Given the evolutive dead-end nature of L. pneumophila infection in humans the study of the pathogenicity of L. pneumophila from natural environments isolates, as well as from man-made environments and clinical samples, is important to determine which factors contribute to the occurrence of disease. Using seven isolates from natural environments, five from man-made environments and four clinical-related L. pneumophila strains, we found that all strains prove to be pathogenic in G. mellonella infection model. In accordance with previous studies (Harding et al., 2012), larvae mortality kinetics and infection profile were strain specific. Indeed, a wide variety of virulence-related phenotypes were observed. While some of the tested strains were defective in their ability to cause disease others were highly pathogenic. Since the L. pneumophila isolates used in this study had distinct virulence-related genetic repertoires (Costa et al., 2010, 2012, 2014) a possible explanation for the above mentioned results could be related to differences in the strains ability to replicate or persist in the infected larvae. Altogether our data could substantiate the existence of significant differences on the ability of $L$. pneumophila strains to induce disease since a correlation has been established between the virulence variation of several human pathogens in G. mellonella model, mammalian cell culture and animal models (Ramarao et al., 2012; Cook and McArthur, 2013; Champion et al., 2016; Tsai et al., 2016; Velikova et al., 2016). The whole genome sequencing of these L. pneumophila natural environmental strains will allow performing genome-wide association studies (GWAs) that will clarify if these variables are statistically dependent. In fact, several GWAs performed in other human pathogens in which differences in genotype frequencies were compared between cases and controls identified novel features related with virulence (Chen and Shapiro, 2015; Omae et al., 2017; Yang et al., 2018).

When L. pneumophila pathogenicity was compared with the origin of the strains no correlation could be established. Indeed, we identified strains capable of inducing $100 \%$ of mortality in G. mellonella isolated from all environmental types. Likewise, strains with a reduced ability to cause death were also isolated from different environments. These findings contradict the idea that isolates of L. pneumophila recovered from clinical cases and man-made environments represent a limited, non-random subset of all genotypes existing in nature with increased capability to induce infection in humans (Harrison et al., 2007; Coscolla and Gonzalez-Candelas, 2009; Costa et al., 2010; David et al., 2016). In addition, we determined that there is a concentration threshold above which L. pneumophila strains are equally able to cause disease in G. mellonella. Overall, our data strongly suggests that the ability of some L. pneumophila strains to cause disease is more likely related with their capability to persist and thrive in man-made environmental niches, which are linked with human infection, and not related with their virulence. This idea is in line with previous reports proposing that natural environments are possible sources or reservoirs of Legionella sp. impacting the spatial distribution of sporadic cases of disease (Ortiz-Roque and Hazen, 1987; Rocha et al., 1995; Costa et al., 2005; Peabody et al., 2017; Cassell et al., 2018).

It is well-documented that the intracellular growth within protozoa is the prevailing mechanism of legionella proliferation (Rowbotham, 1986; Fields, 1996; Boamah et al., 2017; Oliva et al., 2018). Moreover, recent reports determined that the distribution of protozoa between different environmental reservoirs is 
relatively constant (Boamah et al., 2017). Nevertheless, not all protozoa species are hosts of $L$. pneumophila. Thus, the relative abundance of L. pneumophila in different environmental niches may be related with variations in the heterogeneous populations of protozoan hosts and non-hosts (Declerck, 2010; Amaro et al., 2015). Indeed, several factors affect the outcome of legionellaprotozoa interaction, namely the genetic composition of the bacterium since dramatic differences have been reported between strains and species (host tropism), the relative abundance of each organism and the external environment (García et al., 2007; Declerck, 2010; Buse and Ashbolt, 2011; Boamah et al., 2017). Several studies determined that the structure and dynamics of microbial communities may be influenced by abiotic environmental filtering and biotic competition (Winter et al., 2013; Heino et al., 2014; Zhou et al., 2017). We propose that this vision should be applied to the L. pneumophila pathway from natural reservoirs to human infection. Is well-known the distinct distribution pattern of legionellae between reservoirs. So, the ability of legionellae to reside, survive and multiply in biofilm communities that are known to provide protection and nutrients (Taylor et al., 2009; Declerck, 2010; Abdel-Nour et al., 2013; Vatansever and Türetgen, 2015) could represent crucial features for selection by environmental filtering and competition explaining the predominance of some disease-related strains. Indeed, despite the presence of diverse Legionella sp. in natural aquatic reservoirs (Veríssimo et al., 1991; Marrão et al., 1993; Costa et al., 2005; Peabody et al., 2017; Zhang et al., 2017; Cassell et al., 2018), L. pneumophila, namely SG1, is responsible for the vast majority of the reported cases of disease worldwide (Yu et al., 2002; Harrison et al., 2009; Beauté et al., 2013; Beauté and Network on behalf of the E. L. D. S., 2017). However, the environmental distribution of L. pneumophila SG1 (44\%) differs significantly from the distribution of clinical L. pneumophila SG1 strains (Harrison et al., 2007; Kozak et al., 2009; David et al., 2016). In our opinion, environmental filtering and biotic competition represent the most probable evolutive constrains structuring the diversity of L. pneumophila found in artificial environments aiding to explain our results.

Transmission of Legionnaires' disease usually occurs by inhalation of aerosols or aspiration of water containing Legionella

\section{REFERENCES}

Abdelhady, H., and Garduño, R. A. (2013). The progeny of Legionella pneumophila in human macrophages shows unique developmental traits. FEMS Microbiol. Lett. 349, 99-107. doi: 10.1111/1574-6968. 12300

Abdel-Nour, M., Duncan, C., Low, D. E., and Guyard, C. (2013). Biofilms: the stronghold of Legionella pneumophila. Int. J. Mol. Sci. 14, 21660-21675. doi: 10.3390/ijms141121660

Akaike, H. (1974). A new look at the statistical model identification. IEEE Trans. Autom. Control 19, 716-723. doi: 10.1109/TAC.1974.11 00705

Allombert, J., Fuche, F., Michard, C., and Doublet, P. (2013). Molecular mimicry and original biochemical strategies for the biogenesis of a Legionella pneumophila replicative niche in phagocytic cells. Microbes Infect. 15, 981-988. doi: 10.1016/j.micinf.2013.09.007 spp. from point-sources that dispersed contaminated aerosols over a wide area (Mercante and Winchell, 2015). In these chain of events the concentration of Legionella in water systems is a key factor in terms of greatest threat to public health and one should not rely solely on the presence, virulence of the strain and population health status. Indeed our results demonstrate the existence of a concentration threshold above which $L$. pneumophila strains are equally able to cause disease. This implies that the presence of L. pneumophila per si is not synonymous of a threat to public health but reinforces the need to carefully manage the concentration of this bacterium in water distribution systems to avoid reaching a critical threshold. In this way, it is crucial for water distribution systems management and public health to further co-relate Legionella concentration with the probability to cause disease in countries where appropriate surveillance is in place.

\section{AUTHOR CONTRIBUTIONS}

LM, AV, and JC designed the study. PS and IS were in charge of laboratory procedures. PS did the statistical analysis. AV and JC wrote the manuscript. All authors read and approved the final manuscript.

\section{FUNDING}

This work was supported by FCT/MEC through national funds and the co-funding by the FEDER, within the PT2020 Partnership Agreement, and COMPETE 2020, within the project UID/BIA/04004/2013, and Programa Operacional Regional de Lisboa 2020 (LISBOA-01-0145-FEDER-007317). JC and IS acknowledge financial support by postdoctoral grants from Fundação para a Ciência e Tecnologia (FCT) SFRH/BPD/112157/2015 and SFRH/BPD/86475/2012.

\section{SUPPLEMENTARY MATERIAL}

The Supplementary Material for this article can be found online at: https://www.frontiersin.org/articles/10.3389/fcimb. 2018.00097/full\#supplementary-material
Al-Quadan, T., Price, C. T., and Abu Kwaik, Y. (2012). Exploitation of evolutionarily conserved amoeba and mammalian processes by Legionella. Trends Microbiol. 20, 299-306. doi: 10.1016/j.tim.2012.03.005

Amaro, F., Wang, W., Gilbert, J. A., Anderson, O. R., and Shuman, H. A. (2015). Diverse protist grazers select for virulence-related traits in Legionella. ISME J. 9, 1607-1618. doi: 10.1038/ismej.2014.248

Aperis, G., Fuchs, B. B., Anderson, C. A., Warner, J. E., Calderwood, S. B., and Mylonakis, E. (2007). Galleria mellonella as a model host to study infection by the Francisella tularensis live vaccine strain. Microbes Infect. 9, 729-734. doi: 10.1016/j.micinf.2007.02.016

Aurass, P., Schlegel, M., Metwally, O., Harding, C. R., Schroeder, G. N., Frankel, G., et al. (2013). The Legionella pneumophila Dot/Icm-secreted effector $\mathrm{PlcC} / \mathrm{CegC1}$ together with $\mathrm{PlcA}$ and $\mathrm{PlcB}$ promotes virulence and belongs to a novel zinc metallophospholipase $\mathrm{C}$ family present in bacteria and fungi. J. Biol. Chem. 288, 11080-11092. doi: 10.1074/jbc.M112. 426049 
Barnoy, S., Gancz, H., Zhu, Y., Honnold, C. L., Zurawski, D. V., and Venkatesan, M. M. (2017). The Galleria mellonella larvae as an in vivo model for evaluation of Shigella virulence. Gut Microbes 8, 335-350. doi: 10.1080/19490976.2017.1293225

Beauté, J., and Network on behalf of the E. L. D. S. (2017), Legionnaires' disease in Europe, 2011 to 2015. Euro Surveill. 22:30566. doi: 10.2807/1560-7917.ES.2017.22.27.30566

Beauté, J., Zucs, P., de Jong, B., and European Legionnaires Disease Surveillance Network (2013). Legionnaires disease in Europe, 2009-2010. Euro Surveill. 18:20417. doi: 10.2807/ese.18.10.20417-en

Boamah, D. K., Zhou, G., Ensminger, A. W., and O'Connor, T. J. (2017). From many hosts, one accidental pathogen: the diverse protozoan hosts of Legionella. Front. Cell. Infect. Microbiol. 7:477. doi: 10.3389/fcimb.2017.00477

Borges, V., Nunes, A., Sampaio, D. A., Vieira, L., Machado, J., Simões, M. J., et al. (2016). Legionella pneumophila strain associated with the first evidence of person-to-person transmission of Legionnaires' disease: a unique mosaic genetic backbone. Sci. Rep. 6:26261. doi: 10.1038/srep26261

Brenner, D. J., Steigerwalt, A. G., Epple, P., Bibb, W. F., Mckinney, R. M., Starnes, R. W., et al. (1988). Legionella pneumophila serogroup Lansing 3 isolated from a patient with fatal pneumonia, and descriptions of $L$. pneumophila subsp. pneumophila subsp. nov., L. pneumophila subsp. fraseri subsp. nov., and L. pneumophila subsp. pascullei subsp. nov. J. Clin. Microbiol. 26, 1695-1703.

Buchrieser, C. (2011). Legionella: from protozoa to humans. Front. Microbiol. 2:182. doi: 10.3389/fmicb.2011.00182

Buse, H. Y., and Ashbolt, N. J. (2011). Differential growth of Legionella pneumophila strains within a range of amoebae at various temperatures associated with in-premise plumbing. Lett. Appl. Microbiol. 53, 217-224. doi: 10.1111/j.1472-765X.2011.03094.x

Cassell, K., Gacek, P., Warren, J. L., Raymond, P. A., Cartter, M., and Weinberger, D. M. (2018). Association between sporadic legionellosis and river systems in Connecticut. J. Infect. Dis. 217, 179-187. doi: 10.1093/infdis/jix531

Champion, O. L., Karlyshev, A. V., Senior, N. J., Woodward, M., La Ragione, R., Howard, S. L., et al. (2010). Insect infection model for Campylobacter jejuni reveals that O-methyl phosphoramidate has insecticidal activity. J. Infect. Dis. 201, 776-782. doi: 10.1086/650494

Champion, O. L., Wagley, S., and Titball, R. W. (2016). Galleria mellonella as a model host for microbiological and toxin research. Virulence 7, 840-845. doi: 10.1080/21505594.2016.1203486

Chen, P. E., and Shapiro, B. J. (2015). The advent of genome-wide association studies for bacteria. Curr. Opin. Microbiol. 25, 17-24. doi: 10.1016/j.mib.2015.03.002

Chien, M., Morozova, I., Shi, S., Sheng, H., Chen, J., Gomez, S. M., et al. (2004). The genomic sequence of the accidental pathogen Legionella pneumophila. Science 305, 1966-1968. doi: 10.1126/science. 1099776

Cook, S. M., and McArthur, J. D. (2013). Developing Galleria mellonella as a model host for human pathogens. Virulence 4, 350-353. doi: 10.4161/viru.25240

Correia, A. M., Ferreira, J. S., Borges, V., Nunes, A., Gomes, B., Capucho, R., et al. (2016). Probable person-to-person transmission of Legionnaires' Disease. $N$. Engl. J. Med. 374, 497-498. doi: 10.1056/NEJMc1505356

Coscollá, M., Comas, I., and González-Candelas, F. (2011). Quantifying nonvertical inheritance in the evolution of Legionella pneumophila. Mol. Biol. Evol. 28, 985-1001. doi: 10.1093/molbev/msq278

Coscollá, M., and Gonzalez-Candelas, F. (2009). Comparison of clinical and environmental samples of Legionella pneumophila at the nucleotide sequence level. Infect. Genet. Evol. 9, 882-888. doi: 10.1016/j.meegid.2009.05.013

Costa, J., d'Avó, A. F., da Costa, M. S., and Veríssimo, A. (2012). Molecular evolution of key genes for type II secretion in Legionella pneumophila. Environ. Microbiol. 14, 2017-2033. doi: 10.1111/j.1462-2920.2011.02646.x

Costa, J., Teixeira, P. G., d’Avó, A. F., Júnior, C. S., and Veríssimo, A. (2014). Intragenic recombination has a critical role on the evolution of Legionella pneumophila virulence-related effector SidJ. PLoS ONE 9:e109840. doi: 10.1371/journal.pone.0109840

Costa, J., Tiago, I., Da Costa, M. S., and Veríssimo, A. (2005). Presence and persistence of Legionella spp. in groundwater. Appl. Environ. Microbiol. 71, 663-671. doi: 10.1128/AEM.71.2.663-671.2005

Costa, J., Tiago, I., da Costa, M. S., and Veríssimo, A. (2010). Molecular evolution of Legionella pneumophila $\operatorname{dotA}$ gene, the contribution of natural environmental strains. Environ. Microbiol. 12, 2711-2729. doi: 10.1111/j.1462-2920.2010.02240.x

Cunha, B. A., Burillo, A., and Bouza, E. (2016). Legionnaires' disease. Lancet 387, 376-385. doi: 10.1016/S0140-6736(15)60078-2

David, S., Rusniok, C., Mentasti, M., Gomez-Valero, L., Harris, S. R., Lechat, P., et al. (2016). Multiple major disease-associated clones of Legionella pneumophila have emerged recently and independently. Genome Res. 26, 1555-1564. doi: 10.1101/gr.209536.116

Declerck, P. (2010). Biofilms: the environmental playground of Legionella pneumophila. Environ. Microbiol. 12, 557-566. doi: 10.1111/j.1462-2920.2009.02025.x

Doleans, A., Aurell, H., Reyrolle, M., Lina, G., Freney, J., Vandenesch, F., et al. (2004). Clinical and environmental distributions of legionella strains in France are different. J. Clin. Microbiol. 42, 458-460. doi: 10.1128/JCM.42.1.458-460.2004

Eisenman, H. C. (2015). Metamorphosis of Galleria mellonella research. J. Virulence 6, 1-2. doi: 10.1080/21505594.2014.998541

Fields, B. (2008). "Legionella in the environment," in Legionella Pneumophila: Pathogenesis and Immunity, eds M. Hoffman, P. Friedman, and H. Bendinelli (New York, NY: Springer Science and Business Media), 85-94.

Fields, B. S. (1996). The molecular ecology of legionellae. Trends Microbiol. 4, 286-290. doi: 10.1016/0966-842X(96)10041-X

Finsel, I., and Hilbi, H. (2015). Formation of a pathogen vacuole according to Legionella pneumophila : how to kill one bird with many stones. Cell Microbiol. 17, 935-950. doi: 10.1111/cmi.12450

Francisco, A. P., Bugalho, M., Ramirez, M., and Carriço, J. A. (2009). Global optimal eBURST analysis of multilocus typing data using a graphic matroid approach. BMC Bioinformatics 10:152. doi: 10.1186/1471-2105-10-152

Fraser, D. W., Tsai, T. R., Orenstein, W., Parkin, W. E., Beecham, H. J., Sharrar, R. G., et al. (1977). Legionnaires' disease. N. Engl. J. Med. 297, 1189-1197.

Gaia, V., Fry, N. K., Afshar, B., Lü, P. C., Etienne, J., Peduzzi, R., et al. (2005). Consensus sequence-based scheme for epidemiological typing of clinical and environmental isolates of Legionella pneumophila. J. Clin. Microbiol. 43, 2047-2052. doi: 10.1128/JCM.43.5.2047-2052.2005

García, M. T., Jones, S., Pelaz, C., Millar, R. D., and Abu Kwaik, Y. (2007) Acanthamoeba polyphaga resuscitates viable non-culturable Legionella pneumophila after disinfection. Environ. Microbiol. 9, 1267-1277. doi: 10.1111/j.1462-2920.2007.01245.x

Garcia-Vallvé S., and Palau, J. R. A. (1999). Horizontal gene transfer in glycosyl hydrolases inferred from codon usage in Escherichia coli and Bacillus subtilis. Mol. Biol. Evol. 16, 1125-1234. doi: 10.1093/oxfordjournals.molbev.a0 26203

Gomez-Valero, L., and Buchrieser, C. (2013). Genome dynamics in Legionella: the basis of versatility and adaptation to intracellular replication. Cold Spring Harb. Perspect. Med. 3:a009993. doi: 10.1101/cshperspect.a009993

Gomez-Valero, L., Rusniok, C., Jarraud, S., Vacherie, B., Rouy, Z., Barbe, V., et al. (2011). Extensive recombination events and horizontal gene transfer shaped the Legionella pneumophila genomes. BMC Genomics 12:536. doi: 10.1186/1471-2164-12-536

Gomez-Valero, L., Rusniok, C., Rolando, M., Neou, M., Dervins-Ravault, D., Demirtas, J., et al. (2014). Comparative analyses of Legionella species identifies genetic features of strains causing Legionnaires' disease. Genome Biol. 15:505. doi: 10.1186/PREACCEPT-1086350395137407

Guindon, S., and Gascuel, O. (2003). A simple, fast, and accurate algorithm to estimate large phylogenies by maximum likelihood. Syst. Biol. 52, 696-704. doi: 10.1080/10635150390235520

Harding, C. R., Mattheis, C., Mousnier, A., Oates, C. V., Hartland, E. L., Frankel, G., et al. (2013a). LtpD is a novel legionella pneumophila effector that binds phosphatidylinositol 3-phosphate and inositol monophosphatase IMPA1. Infect. Immun. 81, 4261-4270. doi: 10.1128/IAI.01054-13

Harding, C. R., Schroeder, G. N., Reynolds, S., Kosta, A., Collins, J. W., Mousnier, A., et al. (2012). Legionella pneumophila pathogenesis in the Galleria mellonella infection model. Infect. Immun. 80, 2780-2790. doi: 10.1128/IAI.00510-12

Harding, C. R., Stoneham, C. A., Schuelein, R., Newton, H., Oates, C. V., Hartland, E. L., et al. (2013b). The Dot/Icm effector SdhA is necessary for virulence of Legionella pneumophila in Galleria mellonella and A/J Mice. Infect. Immun. 81, 2598-2605. doi: 10.1128/IAI.00296-13 
Harrison, T. G., Afshar, B., Doshi, N., Fry, N. K., and Lee, J. V. (2009). Distribution of Legionella pneumophila serogroups, monoclonal antibody subgroups and DNA sequence types in recent clinical and environmental isolates from England and Wales (2000-2008). Eur. J. Clin. Microbiol. Infect. Dis. 28, 781-791. doi: 10.1007/s10096-009-0705-9

Harrison, T. G., Doshi, N., Fry, N. K., and Joseph, C. A. (2007). Comparison of clinical and environmental isolates of Legionella pneumophila obtained in the UK over 19 years. Clin. Microbiol. Infect. 13, 78-85. doi: 10.1111/j.1469-0691.2006.01558.x

Heino, J., Tolkkinen, M., Pirttilä, A. M., Aisala, H., and Mykrä, H. (2014). Microbial diversity and community-environment relationships in boreal streams. J. Biogeogr. 41, 2234-2244. doi: 10.1111/jbi.12369

Higgins, D. G. (1994). CLUSTAL V: multiple alignment of DNA and protein sequences. Methods Mol. Biol. 25, 307-318. doi: 10.1385/0-89603-276-0:307

Hoffmann, C., Harrison, C. F., and Hilbi, H. (2014). The natural alternative: protozoa as cellular models for Legionella infection. Cell. Microbiol. 16, 15-26. doi: $10.1111 / \mathrm{cmi} .12235$

Hoisington, A. J., Brenner, L. A., Kinney, K. A., Postolache, T. T., and Lowry, C. A. (2015). The microbiome of the built environment and mental health. Microbiome 3:60. doi: 10.1186/s40168-015-0127-0

Hubber, A., and Roy, C. R. (2010). Modulation of host cell function by Legionella pneumophila type IV effectors. Annu. Rev. Cell Dev. Biol. 26, 261-283. doi: 10.1146/annurev-cellbio-100109-104034

Hyland, J. M., Hamlet, N., Saunders, C., Coppola, J., and Watt, J. (2008). Outbreak of Legionnaires' disease in West Fife: Review of environmental guidelines needed. Public Health 122, 79-83. doi: 10.1016/j.puhe.2007.05.005

Isberg, R. R., O'Connor, T. J., and Heidtman, M. (2009). The Legionella pneumophila replication vacuole: making a cosy niche inside host cells. Nat. Rev. Microbiol. 7, 13-24. doi: 10.1038/nrmicro1967

Junqueira, J. C. (2012). Galleria mellonella as a model host for human pathogens: recent studies and new perspectives. Virulence 3, 474-476. doi: 10.4161/viru.22493

Khodr, A., Kay, E., Gomez-Valero, L., Ginevra, C., Doublet, P., Buchrieser, C., et al. (2016). Molecular epidemiology, phylogeny and evolution of Legionella. Infect. Genet. Evol. 43, 108-122. doi: 10.1016/j.meegid.2016.04.033

Kozak, N. A., Benson, R. F., Brown, E., Alexander, N. T., Taylor, T. H., Shelton, B. G., et al. (2009). Distribution of lag-1 alleles and sequence-based types among Legionella pneumophila serogroup 1 clinical and environmental isolates in the United States. J. Clin. Microbiol. 47, 2525-2535. doi: 10.1128/JCM.02410-08

Loh, J. M., Adenwalla, N., Wiles, S., and Proft, T. (2013). Galleria mellonella larvae as an infection model for group A streptococcus. Virulence 4, 419-428. doi: 10.4161/viru. 24930

Luo, Z. Q. (2011). Targeting one of its own: expanding roles of substrates of the Legionella pneumophila Dot/Icm type IV secretion system. Front. Microbiol. 2:31. doi: $10.3389 /$ fmicb.2011.00031

Marrão, G., Veríssimo, A., Bowker, R. G., and Costa, M. D. (1993). Biofilms as major sources of Legionella spp. in hydrothermal areas and their dispersion into stream water. FEMS Microbiol. Ecol. 12, 25-33. doi: 10.1111/j.1574-6941.1993.tb00013.x

McAdam, P. R., Vander Broek, C. W., Lindsay, D. S., Ward, M. J., Hanson, M. F., Gillies, M., et al. (2014). Gene flow in environmental Legionella pneumophila leads to genetic and pathogenic heterogeneity within a Legionnaires' disease outbreak. Genome Biol. 15, 504. doi: 10.1186/PREACCEPT-1675723368141690

McDade, J. E., Shepard, C. C., Fraser, D. W., Tsai, T. R., Redus, M. A., and Dowdle, W. R. (1977). Legionnaires' Disease. N. Engl. J. Med. 297, 1197-1203.

McKinney, R. M., Thacker, L., Harris, P. P., Lewallen, K. R., Hebert, G. A., Edelstein, P. H., et al. (1979). Four serogroups of Legionnaires' disease bacteria defined by direct immunofluorescence. Ann. Intern. Med. 90, 621-624.

Mentasti, M., Underwood, A., Lück, C., Kozak-Muiznieks, N. A., Harrison, T. G., and Fry, N. K. (2014). Extension of the Legionella pneumophila sequence-based typing scheme to include strains carrying a variant of the $\mathrm{N}$ acylneuraminate cytidylyltransferase gene. Clin. Microbiol. Infect. 20, O435O441. doi: 10.1111/1469-0691.12459

Mercante, J. W., and Winchell, J. M. (2015). Current and emerging legionella diagnostics for laboratory and outbreak investigations. Clin. Microbiol. Rev. 28, 95-133. doi: 10.1128/CMR.00029-14

Mil-Homens, D., Rocha, E. P., and Fialho, A. M. (2010). Genome-wide analysis of DNA repeats in Burkholderia cenocepacia J2315 identifies a novel adhesin-like gene unique to epidemic-associated strains of the ET-12 lineage. Microbiology 156, 1084-1096. doi: 10.1099/mic.0.032623-0

Milne, I., Wright, F., Rowe, G., Marshall, D. F., Husmeier, D., and McGuire, G. (2004). TOPALi: software for automatic identification of recombinant sequences within DNA multiple alignments. Bioinformatics 20, 1806-1807. doi: 10.1093/bioinformatics/bth155

Moliner, C., Fournier, P.-E., and Raoult, D. (2010). Genome analysis of microorganisms living in amoebae reveals a melting pot of evolution. FEMS Microbiol. Rev. 34, 281-294. doi: 10.1111/j.1574-6976.2009.00209.x

Muder, R. R., and Yu, V. L. (2002). Infection due to Legionella species other than L. pneumophila. Clin. Infect. Dis. 35, 990-998. doi: 10.1086/ 342884

Muder, R. R., Yu, V. L., and Woo, A. H. (1986). Mode of transmission of Legionella pneumophila. Arch. Intern. Med. 146, 1607-1612. doi: 10.1001/archinte.1986.00360200183030

Nascimento, M., Sousa, A., Ramirez, M., Francisco, A. P., Carriço, J. A., and Vaz, C. (2017). PHYLOViZ 2.0: providing scalable data integration and visualization for multiple phylogenetic inference methods. Bioinformatics 33, 128-129. doi: 10.1093/bioinformatics/btw582

Newton, H. J., Ang, D. K., van Driel, I. R., and Hartland, E. L. (2010). Molecular pathogenesis of infections caused by Legionella pneumophila. Clin. Microbiol. Rev. 23, 274-298. doi: 10.1128/CMR.00052-09

Nygård, K., Werner-Johansen, Ø., Rønsen, S., Caugant, D. A., Simonsen, Ø., Kanestrøm, A., et al. (2008). An outbreak of legionnaires disease caused by long-distance spread from an industrial air scrubber in Sarpsborg, Norway. Clin. Infect. Dis. 46, 61-69. doi: 10.1086/524016

Oliva, G., Sahr, T., and Buchrieser, C. (2018). The life cycle of Legionella pneumophila: cellular differentiation is linked to virulence and metabolism. Front. Cell. Infect. Microbiol. 8:3. doi: 10.3389/fcimb.2018.00003

Omae, Y., Toyo-oka, L., Yanai, H., Nedsuwan, S., Wattanapokayakit, S., Satproedprai, N., et al. (2017). Pathogen lineage-based genome-wide association study identified CD53 as susceptible locus in tuberculosis. J. Hum. Genet. 62, 1015-1022. doi: 10.1038/jhg.2017.82

Ortiz-Roque, C. M., and Hazen, T. C. (1987). Abundance and distribution of Legionellaceae in Puerto Rican waters. Appl. Environ. Microbiol. 53, 2231-2236.

Peabody, M. A., Caravas, J. A., Morrison, S. S., Mercante, J. W., Prystajecky, N. A., Raphael, B. H., et al. (2017). Characterization of Legionella Species from watersheds in British Columbia, Canada. mSphere 2:e0246-17. doi: $10.1128 / \mathrm{mSphere.00246-17}$

Phin, N., Parry-Ford, F., Harrison, T., Stagg, H. R., Zhang, N., Kumar, K., et al. (2014). Epidemiology and clinical management of Legionnaires' disease. Lancet Infect. Dis. 14, 1011-1021. doi: 10.1016/S1473-3099(14)70713-3

Posada, D. (2008). jModelTest: phylogenetic model averaging. Mol. Biol. Evol. 25, 1253-1256. doi: 10.1093/molbev/msn083

Posada, D., and Buckley, T. R. (2004). Model selection and model averaging in phylogenetics: advantages of akaike information criterion and bayesian approaches over likelihood ratio tests. Syst. Biol. 53, 793-808. doi: 10.1080/10635150490522304

Ramarao, N., Nielsen-Leroux, C., and Lereclus, D. (2012). The insect Galleria mellonella as a powerful infection model to investigate bacterial pathogenesis. J. Vis. Exp. e4392. doi: 10.3791/4392

Ratzow, S., Gaia, V., Helbig, J. H., Fry, N. K., and Lück, P. C. (2007). Addition of neuA, the gene encoding $\mathrm{N}$-acylneuraminate cytidylyl transferase, increases the discriminatory ability of the consensus sequence-based scheme for typing Legionella pneumophila serogroup 1 strains. J. Clin. Microbiol. 45, 1965-1968. doi: 10.1128/JCM.00261-07

Richards, A. M., Dwingelo, J. E., Price, C. T., and Kwaik, Y. A. (2013). Cellular microbiology and molecular ecology of Legionella - amoeba interaction. Virulence. 4, 307-314. doi: 10.4161/viru.24290

Rocha, G., Veríssimo, A., Bowker, R., and Bornstein, N. (1995). Relationship between Legionella spp . and antibody titres at a therapeutic thermal spa in Portugal. Epidemiol Infect. 115, 79-88. doi: 10.1017/S0950268800058143

Rowbotham, T. J. (1986). Current views on the relationships between amoebae, legionellae and man. Isr. J. Med. Sci. 22, 678-689.

Sánchez-Busó, L., Comas, I., Jorques, G., and González-Candelas, F. (2014). Recombination drives genome evolution in outbreak-related Legionella pneumophila isolates. Nat. Genet. 46, 1205-1211. doi: 10.1038/ ng.3114 
Sanchez, I., Garcia-Nuñez, M., Ragull, S., Sopena, N., Pedro-Botet, M. L., Estere, M., et al. (2008). Genotypic variability and persistence of Legionella pneumophila PFGE patterns in 34 cooling towers from two different areas. Environ. Microbiol. 10, 395-399. doi: 10.1111/j.1462-2920.2007.01 460.x

Schell, M. A., Lipscomb, L., and DeShazer, D. (2008). Comparative genomics and an insect model rapidly identify novel virulence genes of Burkholderia mallei. J. Bacteriol. 190, 2306-2313. doi: 10.1128/JB.01735-07

Tamura, K., Peterson, D., Peterson, N., Stecher, G., Nei, M., and Kumar, S. (2011). MEGA5: molecular evolutionary genetics analysis using maximum likelihood, evolutionary distance, and maximum parsimony methods. Mol. Biol. Evol. 28, 2731-2739. doi: 10.1093/molbev/msr121

Taylor, M., Ross, K., and Bentham, R. (2009). Legionella, protozoa, and biofilms: interactions within complex microbial systems. Microb. Ecol. 58, 538-547. doi: 10.1007/s00248-009-9514-Z

Thomas, V., and Greub, G. (2010). Amoeba/amoebal symbiont genetic transfers: lessons from giant virus neighbours. Intervirology 53, 254-267. doi: 10.1159/000312910

Tsai, C. J., Loh, J. M., and Proft, T. (2016). Galleria mellonella infection models for the study of bacterial diseases and for antimicrobial drug testing. Virulence 7, 214-229. doi: 10.1080/21505594.2015.1 135289

van Heijnsbergen, E., Schalk, J., a., Euser, S., Brandsema, P. S., den Boer, J. W., and De Roda Husman, A. M. (2015). Confirmed and potential sources of Legionella reviewed. Environ. Sci. Technol. 149, 4797-4815. doi: 10.1021/acs.est.5b00142

van Heijnsbergen, E., van Deursen, A., Bouwknegt, M., Bruin, J. P., de Roda Husman, A. M., and Schalk, J. A. C. (2016). Presence and persistence of viable, clinically relevant Legionella pneumophila bacteria in garden soil in the Netherlands. Appl. Environ. Microbiol. 82, 5125-5131. doi: 10.1128/AEM.00595-16

Vatansever, C., and Türetgen, I. (2015). Survival of biofilm-associated Legionella pneumophila exposed to various stressors. Water Environ. Res. 87, 227-232. doi: 10.2175/106143015X14212658613154

Velikova, N., Kavanagh, K., and Wells, J. M. (2016). Evaluation of Galleria mellonella larvae for studying the virulence of Streptococcus suis. BMC Microbiol. 16, 291. doi: 10.1186/s12866-016-0905-2

Veríssimo, A., Marrão, G., da Silva, F. G., and da Costa, M. S. (1991). Distribution of Legionella spp. in hydrothermal areas in continental portugal and the Island of São Miguel, Azores. Appl Environ Microbiol. 57, 2921-2927.
Veríssimo, A., Vesey, G., Rocha, G. M., Marrão, G., Colbourne, J., Dennis, P. J., et al. (1990). A hot water supply as the source of Legionella pneumophila in incubators of a neonatology unit. Hosp. Infect. 15, 255-263.

Wand, M. E., Müller, C. M., Titball, R. W., and Michell, S. L. (2011). Macrophage and Galleria mellonella infection models reflect the virulence of naturally occurring isolates of B. pseudomallei, B. thailandensis and B. oklahomensis. BMC Microbiol. 17:11. doi: 10.1186/1471-2180-11-11

Winter, C., Matthews, B., and Suttle, C. A. (2013). Effects of environmental variation and spatial distance on bacteria, archaea and viruses in subpolar and arctic waters. ISME J. 7, 1507-1518. doi: 10.1038/ismej. 2013.56

Wojda, I. (2017). Immunity of the greater wax moth Galleria mellonella. Insect Sci. 24, 342-357. doi: 10.1111/1744-7917.12325

Yang, C., Yang, R. F., and Cui, Y. (2018). Bacterial genome-wide association study: methodologies and applications. Yi Chuan Hered. 40, 57-65. doi: 10.16288/j.yczz.17-303

Yu, V. L., Plouffe, J. F., Pastoris, M. C., Stout, J. E., Schousboe, M., Widmer, A., et al. (2002). Distribution of Legionella species and serogroups isolated by culture in patients with sporadic community-acquired Legionellosis : an international collaborative survey. J. Infect. Dis. 186, 127-128. doi: 10.1086/341087

Zhang, L., Li, Y., Wang, X., Shangguan, Z., Zhou, H., Wu, Y., et al. (2017). High prevalence and genetic polymorphisms of Legionella in natural and man-made aquatic environments in Wenzhou, China. Int. J. Environ. Res. Public Health 14:E222. doi: 10.3390/ijerph14030222

Zhou, J., Yang, H., Tang, F., Koide, R. T., Cui, M., Liu, Y., et al. (2017). Relative roles of competition, environmental selection and spatial processes in structuring soil bacterial communities in the Qinghai-Tibetan Plateau. Appl. Soil Ecol. 117-118, 223-232. doi: 10.1016/j.apsoil.2017.05.012

Conflict of Interest Statement: The authors declare that the research was conducted in the absence of any commercial or financial relationships that could be construed as a potential conflict of interest.

Copyright (C) 2018 Sousa, Silva, Moreira, Veríssimo and Costa. This is an open-access article distributed under the terms of the Creative Commons Attribution License (CC $B Y)$. The use, distribution or reproduction in other forums is permitted, provided the original author(s) and the copyright owner are credited and that the original publication in this journal is cited, in accordance with accepted academic practice. No use, distribution or reproduction is permitted which does not comply with these terms. 